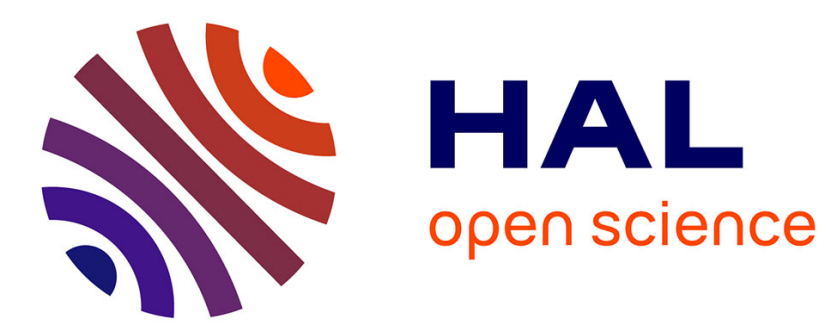

\title{
L'économie politique des séries américaines
}

David Buxton

\section{- To cite this version:}

David Buxton. L'économie politique des séries américaines. Mouvements: des idées et des luttes, 2011, 67, pp.85-91. 10.3917/mouv.067.0085 . hal-01949565

\section{HAL Id: hal-01949565 \\ https://hal.parisnanterre.fr/hal-01949565}

Submitted on 10 Dec 2018

HAL is a multi-disciplinary open access archive for the deposit and dissemination of scientific research documents, whether they are published or not. The documents may come from teaching and research institutions in France or abroad, or from public or private research centers.
L'archive ouverte pluridisciplinaire HAL, est destinée au dépôt et à la diffusion de documents scientifiques de niveau recherche, publiés ou non, émanant des établissements d'enseignement et de recherche français ou étrangers, des laboratoires publics ou privés. 


\section{L'économie politique des séries américaines}

\section{Par David BUXTON *}

* Professeur en sciences de l'information et de la communication à l'université de Paris Ouest Nanterre La Défense. Il a publié Les séries télévisées. Forme, idéologie et mode de production, L'Harmattan, Paris, 2010.

\begin{abstract}
Le genre policier et ses variantes ont dominé la série télévisée depuis les années 1970. C'est l'adaptation au genre des personnages sans profondeur et d'une écriture modulaire, deux inventions formelles de la "série pop " des années 1960, qui a permis aux séries américaines de conquérir le monde entier, au-delà des facteurs économiques. Mais la tendance actuelle à la convergence des supports pourrait menacer à terme la forme série.
\end{abstract}

$\tau$ amais, les séries américaines n’ont été aussi présentes sur les écrans du monde entier ; en France, un véritable travail de revalorisation à leur égard a été entamé dans les médias depuis une dizaine d'années, et relayé depuis peu par la critique universitaire. Cette critique tend à être " culturaliste ", c'est-à-dire que l'on prétend déduire directement des vérités sur la société américaine à partir d'un de ses artefacts. Que certains journalistes (dans le sillage de l'écrivain Martin Winckler) soient enthousiasmés par les séries américaines charrie parfois un plaidoyer lénifiant sur les mérites politiques et des séries et de la société dont elles sont issues, alors que les thèmes "libéraux " (tolérance de la diversité ethnique, de l'homosexualité, féminisme, etc.) sont parfaitement compatibles avec le capitalisme "tardif ". La série fait corps avec la télévision commerciale et ne saurait exister en dehors d'elle. Ainsi, un certain type d'analyse accroît la valeur d'usage de la série américaine, notamment en contribuant à son adaptation, pays par pays, à un marché désormais international. Sans nier la validité (je plaide coupable !) d'une analyse critique de séries particulières, cet article tord le bâton dans l'autre sens en privilégiant la dimension économique, largement ignorée dans les travaux universitaires.

\section{Origines d'un marché international}

La sérialité constitue une des formes de marquage essentielles à la production culturelle à des fins marchandes. Le déplacement du centre de gravité de la télévision américaine de New York à Hollywood vers la fin des années 1950 accéléra l'emprise de la forme série qui pouvait utili- 
ser et réutiliser l'infrastructure des studios. Pour satisfaire les demandes incessantes des programmateurs, il fallut logiquement s'appuyer sur les mêmes acteurs et les mêmes décors de façon régulière. Les séries (dans un premier temps "semi-anthologiques " avec un ou deux personnages récurrents mais des thèmes très variés d'un épisode à l'autre) pouvaient aussi faire un usage plus rationnel des guest stars, alors que les tournages en direct immobilisaient même les petits rôles le temps de la répétition. Une formule se créa : chaque épisode d'une série contenait un nombre limité de personnages réguliers confrontés à des passants qui croisaient leur chemin. Second avantage : les séries étaient plus faciles à manier par les scénaristes, considération importante lors de l'industrialisation de la production. Alors que pour écrire une pièce dramatique, l'auteur doit inventer une intrigue et des personnages originaux, pour un épisode d'une série, il peut partir de personnages préétablis. Mais par-dessus tout, la forme série a permis de résoudre le problème de la régularité de l'audience qui devenait aigu quand les spots publicitaires groupés remplaçaient la sponsorisation directe des émissions. Dès lors que les châ̂nes ont acquis le contrôle financier nécessaire pour imposer leur propre programmation (en alliance avec les sociétés de production), les annonceurs ne furent plus autorisés à intervenir dans le contenu des émissions : en contrepartie, les chaînes devaient leur garantir un public régulier pour le même créneau hebdomadaire, l'espace des spots publicitaires étant vendu à l'avance à des annonceurs divers. La forme série répond aussi aux attentes du consommateur dans un domaine où la valeur d'usage du produit (qui "se paie " en temps investi) est hautement aléatoire.

Les westerns dominaient la télévision américaine à une époque où le marché pour les séries restait plutôt confiné aux frontières nationales. Un marché international pour des produits télévisuels américains s'est mis en place vers la fin des années 1950 ; en 1961, CBS a vendu The Lone Ranger dans 24 pays. Un nouveau type de série, né d'un désir de rompre avec les lourdeurs psychologiques de la série western (en fait du drame contemporain déguisé) a eu sensiblement plus de succès sur le marché international ; Agents très spéciaux (1964-1968) s'est vendu dans 60 pays, Mission impossible (1966-1973) dans 87. Le désir d'être plus présent sur le marché international préside à la genèse de la série Agents très spéciaux: " [le producteur Norman Felton se souvient qu'une responsable de la télévision britannique] lui avait demandé pourquoi dans toutes les séries américaines comme les westerns, tous les héros mesuraient $1 \mathrm{~m} 90$ et étaient forts et costauds... Peut-être devrions-nous penser à une série avec une dimension internationale avec moins de costauds et plus de malins ${ }^{1}$. " Les séries western étaient trop ancrées dans la culture populaire américaine pour être facilement exportables, même dans les pays anglophones. Au-delà des facteurs économiques (notamment la possibilité d'amortir des productions relativement chères sur le marché américain), ce nouveau type de série (" série pop ") avec des personnages sortis d'une bande dessinée, sans profondeur et calqués sur la mode courante, étaient mieux à même de pénétrer les marchés étrangers ; également, elle attirait
1. J. Heitland, The Man from UNCLE Book, St Martin's Press, New York, 1987, p. 4-5. 
2. Les séries produites pour les chaînes câblées (Les Sopranos, The Wire), moins soumises aux taux d'audience, peuvent se contenter d'une douzaine d'épisodes par saison et d'un temps de production moins court, d'où une certaine qualité qui conforte les arguments des "sériephiles".

3. Ibid., p. 188-189 (je traduis). une audience sensiblement plus jeune, plus en phase avec les annonces publicitaires qui désormais s'inséraient à intervalles réguliers.

L'enjeu pour Des Agents très spéciaux était de se rapprocher d'un style cinématographique (en étant moins théâtral) avec des budgets réduits. La nouvelle caméra Arriflex, tenue à la main et fonctionnant à piles, permettait le tournage rapide, sur ces terrains annexes des studios, de scènes d'action normalement trop coûteuses pour une série. La loi d'airain de la production d'une vingtaine d'épisodes par an imposait une organisation rationnelle en trois étapes: préproduction (casting, répétitions, repérages), production (tournage en sept jours) et postproduction (montage, doublage, effets sonores, musique). On pourrait parler d'une production à la chaîne, car à un moment donné, il y a un épisode en préproduction, un en production, et deux ou trois en postproduction (plus trois ou quatre autres en écriture). Ces normes industrielles restent en vigueur aujourd'hui ${ }^{2}$.

\section{Séries et feuilletons}

Qu'est-ce qui change fondamentalement entre les années 1950 et les années 1970, en dehors du contenu manifeste, et en dehors de l'adaptation d'un style plus cinématographique aux contraintes économiques? Après tout, la série d'espionnage des années 1960 a disparu dans la décennie suivante, marquée par le retour en force de la série policière qui depuis, avec ses variantes récentes hospitalières (Dr House), légistes (Les Experts, NCIS, Bones) ou psychologiques (Mentalist, Lie to me), domine la forme au point d'être confondue avec elle. En s'affranchissant du réalisme, psychologique ou social, Des Agents très spéciaux s'exposaient à des scénarios de plus en plus " tirés par les cheveux "; sans un minimum de réalisme, l'écriture sérielle s'avère difficile à maintenir dans la durée, ce qui favorisait le genre policier plus à même de s'inspirer de vrais cas criminels. Lors d'une vaine tentative de renverser la baisse de l'audience, le mémorandum pour scénaristes de la quatrième (et dernière) saison (1967-1968) des Agents très spéciaux a précisé : "il faut bien comprendre qu'à aucun moment l'humour ne devrait gêner l'intrigue ou annuler le suspense... [Les intrigues] doivent être crédibles, conséquentes, "glamoureuses", sophistiquées, avec un rythme rapide, pleines de surprises et entrelacées d'humour... [Les protagonistes Solo et Kuryakin] ne devraient jamais plaisanter alors qu'ils sont en danger ${ }^{3}$ ". De telles consignes sont impossibles à suivre de manière synthétique. Elles impliquent le découpage de l'intrigue en segments modulaires dans lesquels les thèmes divers peuvent être traités tour à tour. La vraie coupure entre les séries "classiques " et les séries " modernes " se trouve là : non pas tant dans l'absence de dimension psychologique (ou morale), mais dans l'extraction de celle-ci d'une intrigue linéaire. Autrement dit, les personnages préétablis peuvent " démontrer " leur personnalité en la compagnie de leurs collègues, amis, partenaires sexuels dans des séquences réservées à cet effet qui n'influent pas nécessairement sur les intrigues en cours. La voie est ouverte à la domination de la série policière à personnages multiples à partir des années 1980. 
À partir de 1969, c'est la " qualité " de l'audience et non la seule quantité qui commence à compter dans les calculs faits par les directeurs des networks. La société Nielsen était désormais en mesure de fournir une corrélation entre la consommation de tel produit et le fait de regarder telle émission, plus une "démographique ", une analyse d'audience spécifique en termes d'âge, sexe, habitation et niveau d'études. Les plus grands consommateurs des produits annoncés à la télévision étaient des habitants de grandes et moyennes villes entre 18 et 49 ans, avec une légère majorité féminine. L'année 1970 a vu en effet une guerre autour de la bonne interprétation des indices entre CBS ("quantité ") et NBC ("qualité "); ce sont les annonceurs qui ont finalement donné raison à la conception "démographique " des indices. Pour les professionnels, le problème de la programmation avait désormais un double aspect : attirer des consommateurs plus jeunes sans se mettre à dos l'audience existante, et attirer plus de femmes pour les émissions prime time sans se mettre à dos le public masculin. La nouvelle donne favorisait plus d'expérimentation, une surproduction de "pilotes " cherchant tous à se démarquer par rapport aux autres (seulement un tiers de la centaine de pilotes tournés annuellement trouvaient leur place dans une grille, et seulement $15 \%$ de ces derniers survivaient plusieurs saisons).

Une des réponses formelles explorées dans les années 1970 fut le tandem de deux policiers, partenaires, mais de personnalités et d'origines radicalement différentes, voire opposées ; on pourrait citer à cet égard Starsky et Hutch (1975-1979) et Miami Vice (1984-1989). Une autre solution fut l'équipe diverse et multiethnique (Hawaï, Police d'État (1968-1980) ; Kojak (1973-1978). Mais c'est dans Hill Street Blues (1981-7), série produite par Steven Bochco, que la plupart de ces innovations s'agrègent sous une forme hybride, à la fois série, feuilleton, comédie de situation, soap opera et cinémavérité. Le commissariat à Hill Street, situé dans une grande ville fictive de la côte est, abrite quatorze personnages récurrents (dont trois femmes), d'origines ethniques diverses, et de tous les bords politiques. Chaque épisode contenait plusieurs intrigues entremêlées, mobilisant plusieurs personnages, qui se poursuivaient d'un épisode à l'autre ; certaines des intrigues dévoilaient la vie privée (alcoolisme, adultères) des policiers, ce qui était nouveau. Le mélange d'éléments sériels et feuilletonnants au sein d'une même saison relève d'un calcul de rentabilité fin. Le feuilleton fidélise davantage et permet des arcs narratifs (des intrigues qui traversent des épisodes, parfois espacés). Il a contre lui le fait qu'on peut difficilement "prendre le train en marche "; la forme se prête moins bien à la rediffusion (syndication) dans la mesure où le déroulement est déjà connu. En revanche, l'intelligibilité de la série classique ne demande pas d'être fidèle chaque semaine ; les épisodes peuvent être rediffusés quotidiennement ou dans le désordre sans perte de sens. 
4. Cité par R. Pearson, "Anatomising Gilbert Grissom ", in M. AlLEN (dir), Reading CSI, op. cit., p. 40 (je traduis).
5. Z. SHERman, cité in "Fox TV looks toward Asia for partners ", Variety, 21 avril 2009 (je traduis).
Alors que les scénaristes préfèrent la forme feuilletonnante qui leur donne un rôle plus stratégique, les producteurs préfèrent une forme mixte qui privilégie les épisodes autonomes. Selon Antony Zuiker, producteur des Experts : "Nous ne voulions pas menotter les téléspectateurs à leurs téléviseurs d'une semaine à l'autre... Ce qui fonctionne le mieux aujourd'hui, ce sont des épisodes autonomes avec des arcs feuilletonnants pour les personnages. [Cette formule] réduit la pression à regarder chaque semaine, mais récompense les téléspectateurs loyaux en mettant en feuilleton les temps forts des personnages. On préfère que vous regardiez 20 ou 24 épisodes plutôt que vous en manquiez deux et abandonniez définitivement ${ }^{4}$ ". Ce qui a changé dans Les Experts (2000), c'est que les multiples personnages récurrents ne sont que faiblement préétablis, étant réduits pour l'essentiel à leur fonction professionnelle. Une back story pour chaque personnage, des éléments biographiques censés révéler leur caractère, est dispensée parcimonieusement et n'a normalement aucune incidence sur le déroulement de l'enquête. Une des conséquences de la série feuilletonnante, c'est l'inflation des coûts "variables" (above the line). En raison de l'augmentation en spirale des salaires des comédiens principaux et de l'équipe "créative" (scénaristes, monteurs) en position de monnayer leurs efforts après le rodage d'une série à succès, celleci porte une charge financière importante à partir de sa cinquième saison, au moment où elle commence à profiter des ventes internationales et en rediffusion. Le comédien Kiefer Sutherland (24) s'est réengagé en 2006 pour trois saisons moyennant 555500 \$ par épisode, alors que le contrat de William Peterson (Les Experts) s'est terminé à 600000 \$ par épisode. Les coûts de production below the line (frais fixes) étant relativement standards pour toutes les séries (entre 100000 \$ et 150000 \$ par jour de tournage), l'inflation vient donc des coûts variables, contrairement à la situation dans les années 1960. À moyen terme, il faudra trouver d'autres montages financiers, en commençant par la coproduction internationale. "Le marché de la télévision est en train de rattraper le business model du cinéma, à savoir, la prévente internationale. Les networks américains ont besoin d'une solution financière pour les séries scénarisées, alors que les acheteurs étrangers ont besoin des produits de qualité qu'ils attendent d'Hollywood ${ }^{5}$."

\section{Coûts de production et audimat}

Les coûts de production ont donc beaucoup augmenté en dollars constants : les 75000 \$ que coûtait un épisode de Cheyenne (1960) correspondent à 470000 \$ en 2008 ; les 155000 \$ d'un épisode des Agents très spéciaux (1965) à 906400 \$; les 400000 \$ de Kojak (1977) à 1,27 \$ million; le 1 million \$ de Miami Vice (1985) à 1,85 million \$, le 1,3 million $\$$ de X-Files (1995) à 1,7 million $\$$, et le 1,8 million $\$$ de CSI (2000) à 2,25 millions \$. Le coût moyen d'une série en prime time en 2007 atteignait 3 millions $\$$. Contraints de vendre aux networks à perte (normalement à 75-80\% des frais), les producteurs dépendent des rediffusions (à partir d'un minimum de 70 épisodes) et des ventes internationales (sans oublier les DVD et les produits dérivés) pour faire des bénéfices. Depuis la grève des scénaristes de télévision à l'hiver 2007-2008, et la crise financière de 2008 , on cherche à réduire le coût par épisode, qui est tout de 
même monté à 5 millions \$ lors de la dernière saison de 24 en 2010, alors que CSI a atteint 4,5 millions $\$$ et NCIS au moins 4 millions $\$$.

Quant aux recettes publicitaires, la marge pour le diffuseur vient de l'extension de l'espace publicitaire. À partir des années 1990, la durée normale d'un épisode passe en conséquence de 52 à 42 minutes, la quantité de spots compensant une stagnation de tarifs aggravée par la croissance de chaînes câblées qui concurrençaient de plus en plus les networks. Les tarifs publicitaires en 2010, qui ont peu évolué depuis 1965 en dollars constants pour la plupart des séries, reflètent l'émiettement de l'audience : une série (et non une sitcom) bien classée aujourd'hui attire une moyenne de 8 à 10 millions de téléspectateurs aux États-Unis par rapport à 20 à 23 millions en 1990 (50 millions pour I Love Lucy dans les années 1950).

Le marché international pour les séries américaines (et depuis le succès de Friends, les sitcoms) s'est considérablement développé depuis les années 1960, aidé par la multiplication de chaînes privées demandeuses de contenus : les trois franchises des Experts ont été vendues dans 200 pays pour une audience cumulée de 2 milliards. À ce titre, l'internationalisation des séries américaines est un bon indice de "l'américanisation " des goûts (en l'occurrence, pour des personnages stéréotypés, dessinés), tendance historique lourde mais toujours réversible : en France, depuis peu, l'audience d'une série américaine dépasse celle d'une fiction française en prime time et le tarif en 2009 pour Les Experts (200 $000 €$ ) constitue une augmentation sensible par rapport à la moyenne pour la diffusion d'un épisode d'une série américaine en France en $2002(60000 \$)^{7}$. Le tarif est fonction du nombre de téléviseurs et de l'importance de l'industrie publicitaire nationale, mais aussi, dans des marchés de taille comparable, du niveau de "résistance " aux séries américaines. Ce dernier semble avoir baissé de manière significative en France dans les années 2000. Une étude macroéconomique des tarifs nationaux des sitcoms américaines en 1983 a conclu que les autres facteurs étant égaux, la proximité culturelle aux États-Unis des pays anglophones fait accrôtre ce tarif par un facteur de deux. Il existait donc historiquement un " discount culturel " dans tous les autres marchés ${ }^{8}$.

Ce qui se profile dans un avenir indéterminé, c'est la convergence des supports : la coexistence de la télévision, des jeux vidéo, de l'informatique et de l'accès à l'internet sur un seul écran, quelle que soit sa taille. Il est probable que la tendance, déjà amorcée, au desserrement des grilles de programmation continuera, et que le consommateur sera de moins en moins contraint de se donner rendez-vous devant l'écran à une heure fixe. Certains acteurs de l'industrie audiovisuelle parlent de la mort inévitable de la télédiffusion commerciale, alors que d'autres préfèrent parier sur la vente directe d'émissions au consommateur en différenciant les prix selon le support (télévision, DVD, téléphone, internet). D’autres encore insistent sur la nécessité d'expérimenter de nouvelles façons d'intégrer la publicité dans le contenu'. Un colloque de professionnels de la télévision en 2009 a entériné la migration vers l'internet des jeunes ; actuellement, même les fans d'une série ne la regardent que la moitié du temps à la télévision $^{10}$. Le modèle d'un financement indirect par la publicité et d'une organisation en grilles programmées pourrait être menacé à terme, et avec lui, la série, du moins sous sa forme actuelle.
6. Les sources diverses pour tous ces chiffres sont données dans mon livre cité dans la notice biographique.

7. $C f$. le coût d'un épisode d'une série policière française : autour de 1,2 million $€$.

8. C. Hoskins, R. Mirus et W. Rozeboom, "US Television programs in the international market: unfair pricing? ", Journal of communication, 39:2, 1989.

9. "Panel weighs TV's future ", Variety, 7 juin 2005.

10. "Milken panel dishes on TV's future ", Variety, 29 avril 2009. 